\title{
Regularity of the Solution of the First Initial-Boundary Value Problem for Hyperbolic Equations in Domains with Cuspidal Points on Boundary
}

\author{
Nguyen Manh Hung ${ }^{1}$ and Vu Trong Luong ${ }^{2}$ \\ ${ }^{1}$ Department of Mathematics, Hanoi National University of Education, Hanoi 4384, Vietnam \\ ${ }^{2}$ Department of Mathematics, Taybac University, Sonla city, Sonla 22384, Vietnam
}

Correspondence should be addressed to Vu Trong Luong, luongvt2003@yahoo.com

Received 3 July 2009; Accepted 8 December 2009

Recommended by Martin Schechter

The goal of this paper is to establish the regularity of the solution of the first initial-boundary value problem for general higher-order hyperbolic equations in cylinders with the bases containing cuspidal points.

Copyright (c) 2009 N. M. Hung and V. T. Luong. This is an open access article distributed under the Creative Commons Attribution License, which permits unrestricted use, distribution, and reproduction in any medium, provided the original work is properly cited.

\section{Introduction}

Initial boundary-value problems for hyperbolic and parabolic type equations in a cylinder with the base containing conical points have been developed sufficiently by us [1-4], the main results of which are about the unique existence of the solution and asymptotic expansions of the solution near a neighborhood of a conical point. However, those problems mentioned above in cylinder with base containing cuspidal point, also interesting for applied sciences, have not been studied yet.

In the present paper, we are concerned with the first initial boundary value problems for higher hyperbolic equation in a cylinder, whose base containing cuspidal points.

In $[5,6]$ we showed the existence of a sequence of smooth domains $\left\{\Omega^{e}\right\}_{\epsilon>0}$ such that $\Omega^{\epsilon} \subset \Omega$ and $\lim _{\epsilon \rightarrow 0} \Omega^{\epsilon}=\Omega$. Furthermore, we proved the existence, the uniqueness, and the smoothness with respect to time variable of the generalized solution by approximating boundary method, which can be applied for nonlinear equations. With the help of the results in $[5,6]$ as well as the results for elliptic boundary value problems in $[7,8]$, we can deal with the regularity with respect to both time variables and spatial ones of the solution. 
Our paper is organized as follows: in Section 2, we introduce exterior cusp domain and weight Sobolev spaces. In Section 3, we will state the formulation of the problem. The main results, Theorems 4.3, 4.6, and 4.7, are stated in Section 4, and examples are given in Section 5.

\section{Cusp Domain and Weighted Sobolev Spaces}

Let $\varphi$ be an infinitely differentiable positive function on the interval $(0,1]$ satisfying the following conditions:

(i) $\lim _{\tau \rightarrow 0} \varphi(\tau)^{k-1} \varphi(\tau)^{(k)}<\infty$ for $k=1,2, \ldots$,

(ii) $\int_{0}^{1}(d \tau / \varphi(\tau))=+\infty$.

These conditions are satisfied; the function $\varphi(\tau)=\tau^{\alpha}$ if $\alpha \geq 1$ is an example. Obviously, conditions (i) and (ii) imply $\varphi(0)=0$. We assume that $\Omega$ is a bounded domain in $\mathbb{R}^{n}, \partial \Omega \backslash\{O\}$ is smooth, and

$$
\left\{x \in \Omega: x_{n}<1\right\}=\left\{x \in \mathbb{R}^{n}: x_{n}<1, x^{\prime} \in \varphi\left(x_{n}\right) \omega\right\},
$$

where $x^{\prime}=\left(x_{1}, \ldots, x_{n-1}\right)$ and $\omega$ is a smooth domain in $\mathbb{R}^{n-1}$. Then the mapping

$$
\begin{gathered}
y_{j}=\frac{x_{j}}{\varphi\left(x_{n}\right)}, \quad j=1, \ldots, n-1, \\
y_{n}=\int_{x_{n}}^{1} \frac{d \tau}{\varphi(\tau)}
\end{gathered}
$$

takes the set $\left\{x \in \Omega: x_{n}<1\right\}$ onto the half-cylinder $\mathcal{C}_{+}=\left\{y \in \mathbb{R}^{n}: y^{\prime} \in \omega, y_{n}>0\right\}=$ $\omega \times(0,+\infty)$. Moreover, it follows that

$$
\operatorname{det}\left(\frac{\partial y_{j}}{\partial x_{k}}\right)_{j, k=1, \ldots, n}=\varphi\left(x_{n}\right)^{-n}
$$

We extend the functions $\varphi$ to an infinitely differentiable positive function on the interval $(0,+\infty)$. The space $H_{\beta, r}^{l}(\Omega)$ can be defined as the closure of the set $C^{\infty}(\bar{\Omega} \backslash\{O\})$ with respect to the norm

$$
\|u\|_{H_{\beta, r}^{l}(\Omega)}=\left(\int_{\Omega|\alpha| \leq l} e^{2 \beta y_{n}\left(x_{n}\right)} \varphi\left(x_{n}\right)^{2(\gamma-l+|\alpha|)}\left|D^{\alpha} u\right|^{2} d x\right)^{1 / 2} .
$$

It is known that $u \in H_{\beta, \gamma}^{l}(\Omega)$, then $\varphi^{k} u \in H_{\beta, \gamma-k}^{l}(\Omega)$ (see [7, Lemma 9.1.4]). 
We also denote by $H^{l}(\Omega)$ the Sobolev space of functions $u=u(x)$ and $x \in \Omega$ that have generalized derivatives $D^{\alpha} u \in L_{2}(\Omega),|\alpha| \leq l$. The norm in this space is defined as follows:

$$
\|u\|_{H^{l}(\Omega)}=\left(\int \sum_{\Omega|\alpha|=0}^{m}\left|D^{\alpha} u\right|^{2} d x\right)^{1 / 2} .
$$

The space $\stackrel{\circ}{H}^{l}(\Omega)$ is the completion of $C_{0}^{\infty}(\Omega)$ in norm of the space $H^{l}(\Omega)$.

Set $Q_{T}=\Omega \times(0, T)$; we proceed to introduce some functional spaces. Let $X, Y$ be Banach spaces, we denote by $L_{2}(0, T ; X)$ the spaces consisting of all measurable functions $u:(0, T) \rightarrow$ $X$ with norm

$$
\|u\|_{L_{2}(0, T ; X)}=\left(\int_{0}^{T}\|u(t)\|_{X}^{2} d t\right)^{1 / 2}
$$

and by $H^{k}(0, T ; X, Y), k=1,2$, the spaces consisting of all functions $u \in L_{2}(0, T ; X)$ such that generalized derivatives $u_{t^{k}}=u^{(k)}$ exist and belong to $L_{2}(0, T ; Y)$, (see [9]), with norms

$$
\|u\|_{H^{k}(0, T ; X, Y)}=\left(\|u\|_{L_{2}(0, T ; X)}^{2}+\sum_{j=1}^{k}\left\|u_{t^{j}}\right\|_{L_{2}(0, T ; Y)}^{2}\right)^{1 / 2}
$$

For shortness, we set

$$
\begin{gathered}
V^{l}(\Omega)=H_{0,0}^{l}(\Omega), \quad V^{l, k}\left(Q_{T}\right)=H^{k}\left(0, T ; V^{l}(\Omega), L_{2}(\Omega)\right), \\
H^{l, 1}\left(Q_{T}\right)=H^{1}\left(0, T ; H^{l}(\Omega), L_{2}(\Omega)\right), \quad H_{\beta, \gamma}^{l, k}\left(Q_{T}\right)=H^{k}\left(0, T ; H_{\beta, \gamma}^{l}(\Omega), L_{2}(\Omega)\right) .
\end{gathered}
$$

Finally, we define the weighted Sobolev space $H_{\beta, \gamma}^{l}\left(Q_{T}\right)$ as a set of all functions defined in $Q_{T}$ such that

$$
\|u\|_{H_{\beta, r}^{l}\left(Q_{T}\right)}=\left(\int_{Q_{T}|\alpha|+k \leq l} e^{2 \beta y\left(x_{n}\right)} \varphi\left(x_{n}\right)^{2(|\gamma-l+\alpha|+k)}\left|D^{\alpha} u_{t^{k}}\right|^{2} d x d t\right)^{1 / 2}<+\infty .
$$

To simplify notation, we continue to write $V^{l}\left(Q_{T}\right)$ instead of $H_{0,0}^{l}\left(Q_{T}\right)$.

\section{Formulation of the Problem}

Let us consider the partial differential operator of order $2 m$

$$
L\left(x, t ; D_{x}\right)=\sum_{|\alpha|,|\beta|=0}^{m} D_{x}^{\alpha}\left(a_{\alpha \beta}(x, t) D_{x}^{\beta}\right)
$$


where $a_{\alpha \beta}$ are functions with complex values, $a_{\alpha \beta}=(-1)^{|\alpha|+|\beta|} a_{\alpha \beta}^{*}\left(a^{*}{ }_{\alpha \beta}\right.$ denotes the transposed conjugate of $a_{\alpha \beta}$ ) and $a_{\alpha \beta}$ are infinitely differentiable in $\bar{Q}_{T}$. Moreover, we assume that the functions

$$
\widehat{a}_{\alpha \beta}(y, \cdot)=\varphi(x(y))^{2 m-|\alpha|-|\beta|} a_{\alpha \beta}(x(y), \cdot)
$$

satisfy the condition of stabilization for $y_{n} \rightarrow+\infty$ for a.e. $t$ in $(0, T)$ (see [7, Section 5.5]). Then the coefficients of the operators $\widehat{L}\left(y, t ; D_{y}\right)$, which arise from operators $\varphi\left(x_{n}\right)^{2 m} L\left(x, t ; D_{x}\right)$ via the coordinate change $x \rightarrow y$, stabilize for $y_{n} \rightarrow+\infty$. If we replace the coefficients of the differential operator $\widehat{L}\left(y, t ; D_{y}\right)$ by their limits for $y_{n} \rightarrow+\infty$, we get differential operator which has coefficients depending only on $y^{\prime}$ and $t$ (for the convenience in use, we denote also by $\left.\widehat{L}\left(y^{\prime}, t ; D_{y^{\prime}}, D_{y_{n}}\right)\right)$.

In the paper, we usually use the following Green's formula:

$$
\int_{\Omega} L\left(x, t ; D_{x}\right) u \bar{v} d x=B(u, v ; t)
$$

which is valid for all $u, v \in C_{0}^{\infty}(\Omega)$ and a.e. $t \in(0, T)$, where

$$
B(u, v ; t)=\sum_{|\alpha|,|\beta|=0}^{m}(-1)^{|\alpha|} \int_{\Omega} a_{\alpha \beta}(\cdot, t) D_{x}^{\beta} u \overline{D_{x}^{\alpha} v} d x
$$

We also suppose that the form $B(\cdot, \cdot ; t)$ is $H^{m}(\Omega)$-elliptic uniformly with respect to $t \in(0, T)$, that is, the inequality

$$
(-1)^{m} B(u, u ; t) \geq \gamma_{0}\|u\|_{H^{m}(\Omega)}^{2}
$$

is valid for all $u \in \stackrel{\circ}{H}^{m}(\Omega)$ and all $t \in(0, T)$, where $\gamma_{0}$ is the positive constant independent of $u$ and $t$. In this paper, we consider the following problem:

$$
\begin{gathered}
(-1)^{m-1} L u-u_{t t}=f \quad \text { in } Q_{T}, \\
u=0, \quad u_{t}=0 \quad \text { on } \Omega, \\
\partial_{v}^{j} u=0 \quad \text { on } S_{T}, \quad j=0,1, \ldots, m-1,
\end{gathered}
$$

where $f \in L_{2}\left(Q_{T}\right)$ and $\partial_{v}^{j} u$ are derivatives with respect to the outer unit normal of $S_{T}=$ $\partial \Omega \backslash\{O\} \times(0, T)$. 
Definition 3.1. A function $u$ is called a generalized solution of problem (3.6)-(3.8) if and only if $u$ belongs to $\stackrel{\circ}{H}^{m, 1}\left(Q_{T}\right), u(x, 0)=0$, and the equality

$$
(-1)^{m-1} \sum_{|\alpha|,|\beta|=0}^{m}(-1)^{|\alpha|} \int_{Q_{T}} a_{\alpha \beta} D_{x}^{\beta} u \overline{D_{x}^{\alpha} \eta} d x d t+\int_{Q_{T}} u_{t} \bar{\eta}_{t} d x d t=\int_{Q_{T}} f \bar{\eta} d x d t
$$

holds for all $\eta \in \stackrel{\circ}{H}^{m, 1}\left(Q_{T}\right), \eta(x, T)=0$.

The existence, the uniqueness and the smoothness with respect to the time variable for the generalized solution of problem (3.6)-(3.8) in the Sobolev space $\stackrel{\circ}{H}^{m, 1}\left(Q_{T}\right)$ were established in $[5,6]$ according to following theorems:

Theorem 3.2. Assume that $f \in L_{2}\left(Q_{T}\right)$, and there exists a positive number $\mu$ such that

$$
\sup \left\{\left|\frac{\partial a_{\alpha \beta}}{\partial t}\right|,\left|a_{\alpha \beta}\right|:(x, t) \in Q_{T}, 0 \leq|\alpha|,|\beta| \leq m\right\} \leq \mu
$$

Then problem (3.6), (3.8) has the unique generalized solution $u \in H^{m, 1}\left(Q_{T}\right)$, and the following estimate holds

$$
\|u\|_{m, 1}^{2} \leq C\|f\|_{L_{2}\left(Q_{T}\right)^{\prime}}^{2}
$$

where $C$ is a constant independent of $u$ and $f$.

Theorem 3.3. Suppose that the following hypotheses are satisfied:

(i) $\partial^{k} f / \partial t^{k} \in L_{2}\left(Q_{T}\right), k \leq h$;

(ii) $\partial^{k} f /\left.\partial t^{k}\right|_{t=0}=0, x \in \Omega, k \leq h-1$;

(iii) $\sup \left\{\left|\partial^{k} a_{\alpha \beta} / \partial t^{k}\right|, k<h:(x, t) \in Q_{T}, 0 \leq|\alpha|,|\beta| \leq m\right\} \leq \mu$.

Then the generalized solution $u \in H^{m, 1}\left(Q_{T}\right)$ of problem (3.6), (3.8) has generalized derivatives with respect to $t$ up to order $h$ in $H^{m, 1}\left(Q_{T}\right)$ and satisfies the following estimate:

$$
\left\|u_{t^{h}}\right\|_{m, 1}^{2} \leq C \sum_{k=0}^{h}\left\|f_{t^{k}}\right\|_{L_{2}\left(Q_{T}\right)^{\prime}}^{2}
$$

where $C$ is a constant independent of $u$ and $f$.

Owing to the support of the following proposition, we can apply the results of the Dirichlet problem for elliptic equation in domains with exterior cusps. 
Proposition 3.4. Suppose that $u=u(x, t)$ is a generalized solution of problem (3.6)-(3.8) and $u_{t t} \in$ $L_{2}\left(Q_{T}\right)$. Then for a.e. $t \in(0, T), u(t)=u(\cdot, t)$ is a generalized solution in $\stackrel{\circ}{H}^{m}(\Omega)$ of the Dirichlet problem for elliptic equation

$$
L\left(\cdot, t D_{x}\right) u=f_{1}(\cdot, t),
$$

where $f_{1}=(-1)^{m-1}\left(u_{t t}+f\right)$.

Proof. Let $\left\{\psi_{k}\right\}_{k=1}^{\infty}$ be an orthogonal basis of the space $\stackrel{\circ}{H}^{m}(\Omega)$. Setting $\eta(x, t)=\psi_{k}(x) \theta(t)$, where $\theta \in C_{0}^{\infty}(0, T)$, and substituting the function $\eta(x, t)$ into (3.9), we conclude that

$$
\int_{Q_{T}}\left[\sum_{|\alpha|,|\beta|=0}^{m}(-1)^{|\alpha|} a_{\alpha \beta} D_{x}^{\beta} u \overline{D_{x}^{\alpha} \psi_{k}}+(-1)^{m}\left(u_{t t} \overline{\psi_{k}}+f \overline{\psi_{k}}\right)\right] \theta(t) d x d t=0 .
$$

We will denote by

$$
\xi(t)=\int_{\Omega}\left[\sum_{|\alpha|,|\beta|=0}^{m}(-1)^{|\alpha|} a_{\alpha \beta} D_{x}^{\beta} u \overline{D_{x}^{\alpha} \psi_{k}}+(-1)^{m}\left(u_{t t} \overline{\psi_{k}}+f \overline{\psi_{k}}\right)\right] d x
$$

that $\xi(t) \in L_{2}(0, T)$. Noting that $\theta \in C_{0}^{\infty}(0, T)$ and using Fubini's theorem, we obtain from (3.14) that $\xi=0$ in $(0, T) \backslash E_{k}$, where $E_{k}$ is a set of measure zero. Since $\left\{\psi_{k}\right\}_{k=1}^{\infty}$ are dense in $\stackrel{\circ}{H}^{m}(\Omega)$, the following equality

$$
\int_{\Omega|\alpha|,|\beta|=0} \sum^{m}(-1)^{|\alpha|} a_{\alpha \beta} D_{x}^{\beta} u \overline{D_{x}^{\alpha} \psi} d x=(-1)^{m-1} \int_{\Omega}\left(u_{t t}+f\right) \bar{\psi} d x
$$

holds for all $\psi \in \stackrel{\circ}{H}^{m}(\Omega)$, for all $t \in(0, T) \backslash \bigcup_{k=1}^{\infty} E_{k}$. It follows that $u(t)$ is a generalized solution in $\stackrel{\circ}{H}^{m}(\Omega)$ of the Dirichlet problem for elliptic equation (3.13), for a.e. $t \in(0, T)$.

\section{The Main Results}

In this section, we would like to present the main results of the study which is based on our previous results (cf. $[5,6])$ and the results of elliptic equations in cusp domains (cf. [7]). For the start of this section, we denote by $\mathcal{U}(\lambda, t)(\lambda \in \mathbb{C}, t \in(0, T))$ the operator corresponding to the parameter-depending boundary value problem

$$
\widehat{L}\left(y^{\prime}, t ; D_{y^{\prime}}, \lambda\right) u=0 \quad \text { in } \omega ; \quad \partial_{v}^{j} u=0 \quad \text { on } \partial \omega, j=1, \ldots, m-1 .
$$

For each $t \in(0, T)$, we have the operator pencil $\mathcal{U}(\lambda, t)$ to be Fredholm, and its spectrum consists of a countable number of isolated eigenvalues. Similarly to Theorem 9.1.4 in [7], we have the following lemma. 
Lemma 4.1. Assume that $f_{1} \in H_{\beta, \gamma}^{k}(\Omega)$, where $\beta, \gamma$ are real numbers. Additionally, the authors suppose that no eigenvalues of $\mathcal{U}(\lambda, t), t \in(0, T)$ line in strips $\operatorname{Re} \lambda_{-} \leq \operatorname{Re} \lambda \leq \operatorname{Re} \lambda_{+}$and $\operatorname{Re} \lambda_{-}<$ $\beta<\operatorname{Re} \lambda_{+}$, where $\lambda_{+}$and $\lambda_{-}$are eigenvalues of $\mathcal{U}(\lambda, t)$, and $\operatorname{Re} \lambda_{-}<0<\operatorname{Re} \lambda_{+}$. Then the generalized solution $u$ of the Dirichlet problem for elliptic equation (3.13), such that $u \equiv 0$ if $x_{n}>1$, belongs to the $H_{\beta, \gamma}^{2 m+k}(\Omega)$ and satisfies the inequality

$$
\|u\|_{H_{\beta, \gamma}^{2 m+k}(\Omega)}^{2} \leq C\left\|f_{1}\right\|_{H_{\beta, \gamma}^{k}(\Omega)^{\prime}}^{2}
$$

where the constant $C$ is independent of $f_{1}$.

Proof. Setting

$$
\omega_{\tau}=\varphi(\tau) \omega
$$

by the Friederichs inequality, we have

$$
\int_{\omega_{\tau}}|u|^{2} d x^{\prime} \leq C \varphi(\tau)^{2 k} \sum_{|r|=k} \int_{\omega_{\tau}}\left|D_{x^{\prime}}^{\gamma} u\right|^{2} d x^{\prime}
$$

therefore,

$$
\varphi\left(x_{n}\right)^{2(|r|-m)} \int_{\omega_{x_{n}}}\left|D_{x^{\prime}}^{\gamma} u\right|^{2} d x^{\prime} \leq C \sum_{|\alpha|=m} \int_{\omega_{x_{n}}}\left|D_{x^{\prime}}^{\alpha} u\right|^{2} d x^{\prime}
$$

for all $|\gamma| \leq m$. Hence,

$$
\sum_{|\gamma| \leq m} \int_{\Omega} \varphi\left(x_{n}\right)^{2(|r|-m)}\left|D_{x}^{\gamma} u\right|^{2} d x \leq C \sum_{|\alpha| \leq m} \int_{\Omega}\left|D_{x}^{\alpha} u\right|^{2} d x
$$

Let $v=v(y)$ be the function which arises from $\varphi\left(x_{n}\right)^{m-n / 2} u(x)$ via the coordinate change $x \rightarrow y$. We set $\bar{\varphi}\left(y_{n}\right)=\varphi\left(x_{n}\right)$; then from the properties of the mapping (2.2) and from inequality (4.6), it follows that $(\bar{\varphi})^{-m+n / 2} v \in H^{m}\left(\mathcal{C}_{+}\right)$. Since $(\bar{\varphi})^{-m+n / 2} v$ is the solution of an elliptic equation in $\mathcal{C}_{+}$with coefficients which stabilize for $y_{n} \rightarrow+\infty$, that is,

$$
\widehat{L}(\bar{\varphi})^{-m+n / 2} v=\widehat{f}_{1}
$$

where $\widehat{f}_{1}=(\bar{\varphi})^{2 m} f_{1}$, we obtain $(\bar{\varphi})^{-m+n / 2} v \in H^{2 m+k}\left(\mathcal{C}_{+}\right)$(cf. [7, Lemma 5.5.3]). This implies $u \in H_{0, m+k}^{2 m+k}(\Omega)$. Using the fact that

$$
\varphi\left(x_{n}\right)^{\gamma-m+k} e^{-\epsilon y_{n}\left(x_{n}\right)} \longrightarrow 0
$$

as $x_{n} \rightarrow 0$, if $0<\epsilon<\beta$, we conclude that $u \in H_{-\epsilon, \gamma}^{2 m+k}(\Omega)$. From Corollary 9.1.1 in [7] it follows that $u \in H_{\beta, \gamma}^{2 m+k}(\Omega)$. Furthermore, (4.2) holds. 
Lemma 4.2. Suppose that $f, f_{t} \in L_{2}\left(Q_{T}\right), f(x, 0)=0$, and the strip $\operatorname{Re} \lambda_{-} \leq \operatorname{Re} \lambda \leq \operatorname{Re} \lambda_{+}$does not contain eigenvalues of $\mathcal{U}(\lambda, t), t \in(0, T)$. Then the generalized solution $u$ of problem (3.6)-(3.8), such that $u \equiv 0$ if $x_{n}>1$, belongs to the $V^{2 m, 2}\left(Q_{T}\right)$ and satisfies the inequality

$$
\|u\|_{V^{2 m, 2}\left(Q_{T}\right)}^{2} \leq C\left[\|f\|_{L_{2}\left(Q_{T}\right)}^{2}+\left\|f_{t}\right\|_{L_{2}\left(Q_{T}\right)}^{2}\right]
$$

where the constant $C$ is independent of $f$.

Proof. Using the smoothness of the generalized solution of problem (3.6)-(3.8) with respect to $t$ in Theorem 3.3 and Proposition 3.4, we can see that for a.e. $t \in(0, T), u \in \stackrel{\circ}{H}^{m}(\Omega)$ is the generalized solution of Dirichlet problem for (3.13) with $f_{1}=u_{t t}+f \in L_{2}(\Omega)=H_{0,0}^{0}(\Omega)=$ $V^{0}(\Omega)$. From Lemma 4.1, it implies that $u \in V^{2 m}(\Omega)$ for a.e. $t \in(0, T)$ and satisfies the inequality

$$
\|u\|_{V^{2 m}(\Omega)}^{2} \leq C_{1}\left\|f_{1}\right\|_{L_{2}(\Omega)}^{2} \leq C\left(\|f\|_{L_{2}(\Omega)}^{2}+\left\|u_{t t}\right\|_{L_{2}(\Omega)}^{2}\right)
$$

By integrating the inequality above with respect to $t$ from 0 to $T$, and using the estimates for derivatives of $u$ with respect to $t$ again, we obtain $u \in V^{2 m, 2}\left(Q_{T}\right)$, which satisfies inequality (4.9).

Theorem 4.3. Let the assumptions of Lemma 4.2 be satisfied, and $f_{t^{k}} \in L_{2}\left(Q_{T}\right), k \leq 2 m, f_{t^{k}}(x, 0)=$ 0 , for $k=0,1, \ldots, 2 m-1$. Then the generalized solution $u$ of problem (3.6)-(3.8), such that $u \equiv 0$ if $x_{n}>1$, belongs to the $V^{2 m}\left(Q_{T}\right)$ and satisfies the inequality

$$
\|u\|_{V^{2 m}\left(Q_{T}\right)}^{2} \leq C \sum_{k=0}^{2 m}\left\|f_{t^{k}}\right\|_{L_{2}\left(Q_{T}\right)}^{2}
$$

where the constant $C$ is independent of $f$.

Proof. Let us first prove that $u_{t^{s}}$ belong to the $V^{2 m, 0}\left(Q_{T}\right)$ for $s=0, \ldots, 2 m-1$ and satisfy

$$
\left\|u_{t^{s}}\right\|_{V^{2 m, 0}\left(Q_{T}\right)}^{2} \leq C \sum_{k=0}^{2 m}\left\|f_{t^{k}}\right\|_{L_{2}\left(Q_{T}\right)}^{2} .
$$

The proof is an induction on $s$. According to Lemma 4.2, it is valid for $s=0$. Now let this assertion be true for $s-1$; we will prove that this also holds for $s$. Due to Lemma 4.2, $u$ satisfies (3.6). By differentiating both sides of (3.6) with respect to $t, s$ times, we obtain

$$
L u_{t^{s}}=(-1)^{m-1}\left(f_{t^{s}}+u_{t^{s+2}}\right)+(-1)^{m} \sum_{k=1}^{s}\left(\begin{array}{l}
s \\
k
\end{array}\right) L_{t^{k}} u_{t^{s-k}},
$$


where

$$
L_{t^{k}}=L_{t^{k}}\left(x, t ; D_{x}\right)=\sum_{\alpha, \beta=0}^{m} D_{x}^{\alpha}\left(\frac{\partial^{k} a_{\alpha \beta}(x, t)}{\partial t^{k}} D_{x}^{\beta}\right) .
$$

By the supposition of the theorem and the inductive assumption, the right-hand side of (4.13) belongs to $L_{2}\left(Q_{T}\right)$. By the arguments analogous to the proof of Lemma 4.2, we get $u_{t^{s}} \in V^{2 m, 0}\left(Q_{T}\right)$ and

$$
\left\|u_{t^{s}}\right\|_{V^{2 m, 0}\left(Q_{T}\right)}^{2} \leq C \sum_{k=0}^{2 m}\left\|f_{t^{k}}\right\|_{L_{2}\left(Q_{T}\right)^{\prime}}^{2}
$$

where $C$ is a constant independent of $u, f$, and $s \leq m-1$.

By using (4.15) and estimates for derivatives of $u$ with respect to $t$ in Theorem 3.3, we have

$$
\begin{aligned}
\|u\|_{V^{2 m}\left(Q_{T}\right)}^{2} & \leq \sum_{k=0}^{2 m-1}\left\|u_{t^{k}}\right\|_{V^{2 m, 0}\left(Q_{T}\right)}^{2}+\left\|u_{t^{2 m}}\right\|_{L_{2}\left(Q_{T}\right)}^{2} \\
& \leq C \sum_{k=0}^{2 m}\left\|f_{t^{k}}\right\|_{L_{2}\left(Q_{T}\right)}^{2} .
\end{aligned}
$$

Remark 4.4. Let $\beta$ be a sufficiently small positive number. Suppose that $e^{\beta y_{n}\left(x_{n}\right)} f \in L_{2}\left(Q_{T}\right)$, and the strip $\operatorname{Re} \lambda_{-} \leq \operatorname{Re} \lambda \leq \operatorname{Re} \lambda_{+}$contains no eigenvalues of $U(\lambda, t), t \in(0, T)$ ); then the generalized solution $u$ of problem (3.6)-(3.8), such that $u \equiv 0$ if $x_{n}>1$, belongs to the $H_{\beta, 0}^{2 m}\left(Q_{T}\right)$. In fact, setting $u=e^{-\beta y_{n}\left(x_{n}\right)} U$, we obtain the first initial boundary value problem which differs little from (3.6)-(3.8). Therefore, $U \in V^{2 m}\left(Q_{T}\right)$, and then $u \in H_{\beta, 0}^{2 m}\left(Q_{T}\right)$. Using the remark above and Lemma 4.1, we obtain the following theorem.

Theorem 4.5. Let the assumptions of Lemma 4.1 be satisfied. Furthermore, the authors assume that $f_{t^{k}} \in H_{\beta, \gamma}^{0}\left(Q_{T}\right), k \leq 2 m$, and $f_{t^{k}}(x, 0)=0$, for $k=0,1, \ldots, 2 m-1$. Then the generalized solution $u$ of problem (3.6)-(3.8), such that $u \equiv 0$ if $x_{n}>1$, belongs to the $H_{\beta, r}^{2 m}\left(Q_{T}\right)$ and satisfies the inequality

$$
\|u\|_{H_{\beta, \gamma}^{2 m}\left(Q_{T}\right)}^{2} \leq C \sum_{k=0}^{2 m}\left\|f_{t^{k}}\right\|_{H_{\beta, r}^{0}\left(Q_{T}\right)^{\prime}}^{2}
$$

where the constant $C$ is independent of $f$.

This theorem is proved by arguments analogous to those proofs of Lemma 4.2 and Theorem 4.3. Next, we will prove the well regularity of the generalized solution of problem (3.6)-(3.8). 
Theorem 4.6. Let the assumptions of Lemma 4.1 be satisfied. Furthermore, the authors assume that $f_{t^{k}} \in H_{\beta, \gamma}^{h}\left(Q_{T}\right), k \leq 2 m+h$, and $f_{t^{k}}(x, 0)=0$, for $k=0,1, \ldots, 2 m+h-1, h \in \mathbb{N}$. Then the generalized solution $u$ of problem (3.6)-(3.8), such that $u \equiv 0$ if $x_{n}>1$, belongs to the $H_{\beta, r}^{2 m+h}\left(Q_{T}\right)$ and satisfies the inequality

$$
\|u\|_{H_{\beta, r}^{2 m+h}\left(Q_{T}\right)}^{2} \leq C \sum_{k=0}^{2 m}\left\|f_{t^{k}}\right\|_{H_{\beta, \gamma}^{h}\left(Q_{T}\right)^{\prime}}^{2}
$$

where the constant $C$ is independent of $u$ and $f$.

Proof. The theorem is proved by induction on $h$. Thanks to Theorem 4.5, this theorem is obviously valid for $h=0$. Assume that the theorem is true for $h-1$, we will prove that it also holds for $h$. It is only needed to show that

$$
\begin{gathered}
u_{t^{s}} \in H_{\beta, \gamma}^{2 m+h-s, 0}\left(Q_{T}\right) \quad \text { for } s=h, h-1, \ldots, 0, \\
\left\|u_{t^{s}}\right\|_{H_{\beta, \gamma}^{2 m+h-s}\left(Q_{T}\right)}^{2} \leq C \sum_{k=0}^{2 m}\left\|f_{t^{k}}\right\|_{H_{\beta, \gamma}^{h}\left(Q_{T}\right)^{2}}^{2}
\end{gathered}
$$

Differentiating both sides of (3.6) again with respect to $t, h$ times, we obtain

$$
L \boldsymbol{u}_{t^{h}}=(-1)^{m-1}\left(f_{t^{h}}+u_{t^{h+2}}\right)+(-1)^{m} \sum_{k=1}^{h}\left(\begin{array}{l}
h \\
k
\end{array}\right) L_{t^{k}} \mathcal{u}_{t^{h-k}} .
$$

By the supposition of the theorem and the inductive assumption, the right-hand side of (4.20) belongs to $H_{\beta, \gamma}^{0}(\Omega)$ for a.e. $t \in(0, T)$. Using Lemma 4.1, we conclude that $u_{t^{h}} \in H_{\beta, \gamma}^{2 m, 0}\left(Q_{T}\right)$. It implies that (4.19) holds for $s=h$. Suppose that (4.19) is true for $s=h, h-1, \ldots, j+1$, and set $v=u_{t^{j}}$, we obtain

$$
L v=F_{j}
$$

where $F_{j}=(-1)^{m-1}\left(f_{t^{j}}+v_{t t}\right)+(-1)^{m} \sum_{k=1}^{j}\left(\begin{array}{l}j \\ k\end{array}\right) L_{t^{k}} u_{t^{j-k}}$. By the inductive assumption with respect to $s, v_{t t}$ belongs to $H_{\beta, \gamma}^{h-j}(\Omega)$ for a.e. $t \in(0, T)$. Thus, the right-hand side of (4.21) belongs to $H_{\beta, \gamma}^{h-j}(\Omega)$. Applying Lemma 4.1 again for $k=h-j$, we get that $v=u_{t j} \in H_{\beta, \gamma}^{2 m+h-j}(\Omega)$ for a.e. $t \in(0, T)$. It means that $v=u_{t^{j}}$ belongs to $H_{\beta, r}^{2 m+h-j, 0}\left(Q_{T}\right)$. Furthermore, we have

$$
\|v\|_{H_{\beta, \gamma}^{2 m+h-j, 0}\left(Q_{T}\right)}^{2} \leq C\left\|F_{j}\right\|_{H_{\beta, \gamma}^{h-j, 0}\left(Q_{T}\right)} \leq C \sum_{k=0}^{2 m}\left\|f_{t^{k}}\right\|_{H_{\beta, \gamma}^{h}\left(Q_{T}\right)}^{2} .
$$


Therefore,

$$
\begin{aligned}
\left\|u_{t^{j}}\right\|_{H_{\beta, \gamma}^{2 m+h-j}\left(Q_{T}\right)}^{2} & \leq\left\|u_{t^{j+1}}\right\|_{H_{\beta, \gamma}^{2 m+h-j-1}\left(Q_{T}\right)}^{2}+\left\|u_{t^{j}}\right\|_{H_{\beta, \gamma}^{2 m+h-j, 0}\left(Q_{T}\right)}^{2} \\
& \leq C \sum_{k=0}^{2 m}\left\|f_{t^{k}}\right\|_{H_{\beta, \gamma}^{h}\left(Q_{T}\right)}^{2} .
\end{aligned}
$$

It implies that (4.19) holds for $s=j$. The proof is complete.

Now we will prove the global regularity of the solution.

Theorem 4.7. Let the hypotheses of Lemma 4.1 be satisfied. Furthermore, suppose $f_{t^{k}} \in H_{\beta, \gamma}^{h}\left(Q_{T}\right)$, $k \leq 2 m+h$, and $f_{t^{k}}(x, 0)=0$,for $k=0,1, \ldots, 2 m+h-1, h \in \mathbb{N}$. Then the generalized solution $u$ of problem (3.6)-(3.8) belongs to the $H_{\beta, r}^{2 m+h}\left(Q_{T}\right)$ and satisfies the inequality

$$
\|u\|_{H_{\beta, r}^{2 m+h}\left(Q_{T}\right)}^{2} \leq C \sum_{k=0}^{2 m}\left\|f_{t^{k}}\right\|_{H_{\beta, r}^{h}\left(Q_{T}\right)^{\prime}}^{2}
$$

where the constant $C$ is independent of $u$ and $f$.

Proof. We denote by $B$ the unit ball, and suppose that $\zeta \in C_{0}^{\infty}(B)$, and $\zeta \equiv 1$ in the neighborhood of the origin $O$. It is easy to get that

$$
(-1)^{m-1} L(\zeta u)-(\zeta u)_{t t}=\zeta f+L_{1} u
$$

where $L_{1}$ is a differential operator, whose coefficients have compact support in a neighborhood of the origin. By arguments analogous to the proof of Theorem 4.6, we obtain

$$
\|\zeta u\|_{H_{\beta, r}^{2 m+h}\left(Q_{T}\right)}^{2} \leq C \sum_{k=0}^{2 m}\left\|f_{t^{k}}\right\|_{H_{\beta, \gamma}^{h}\left(Q_{T}\right)}^{2} .
$$

Set $\zeta_{1} u=(1-\zeta) u$, then $\zeta_{1} u \equiv 0$ in a neighborhood of the origin and $u=\zeta u+(1-\zeta) u$, and using the smoothness of the solution of this problem in domain with smooth boundary, we get

$$
\left\|\zeta_{1} u\right\|_{H^{2 m+h}\left(Q_{T}\right)}^{2} \sim\left\|\zeta_{1} u\right\|_{H_{\beta, r}^{2 m+h}\left(Q_{T}\right)}^{2} \leq C \sum_{k=0}^{2 m}\left\|f_{t^{k}}\right\|_{H_{\beta, r}^{h}\left(Q_{T}\right)}^{2} .
$$

The proof is complete. 


\section{Examples}

In this section, we apply the results of the previous section to the Cauchy-Dirichlet problem for the wave equation. The assumptions can be described as follows: $\Omega$ is a bounded domain in $\mathbb{R}^{n}, \partial \Omega \backslash\{O\}$ is smooth,

$$
\left\{x \in \Omega: 0<x_{n}<1\right\} \equiv\left\{x \in \mathbb{R}^{n}: 0<x_{n}<1,\left|x^{\prime}\right|<\varphi\left(x_{n}\right)\right\}
$$

where $x^{\prime}=\left(x_{1}, \ldots, x_{n-1}\right), \varphi \in C^{\infty}[0,1), \varphi^{\prime}\left(x_{n}\right) \rightarrow 0, \varphi\left(x_{n}\right) \varphi^{\prime \prime}\left(x_{n}\right) \rightarrow 0$ as $x_{n} \rightarrow 0$ and $\varphi(0)=0$, and $Q_{T}=\Omega \times(0, T), S_{T}=\partial \Omega \backslash\{O\} \times(0, T)$.

We consider the Cauchy-Dirichlet problem for the wave equation in $Q_{T}$ :

$$
\begin{gathered}
\Delta u-u_{t t}=f \quad \text { in } Q_{T}, \\
u=0, \quad u_{t}=0 \quad \text { on } \Omega, \\
u=0 \quad \text { on } S_{T}, j=0,1, \ldots, m-1,
\end{gathered}
$$

where $f \in H_{\beta, \gamma}^{0}\left(Q_{T}\right)$. It follows the results of Section 4 that if $|\beta|<z_{+}$, where $z_{+}$is the least positive root of the Bessel function $J_{(n-3) / 2}(z)$, then problem (5.2) has a unique solution $u$ in $H_{\beta, \gamma}^{2}\left(Q_{T}\right)$ and we have the estimate

$$
\|u\|_{H_{\beta, \gamma}^{2}\left(Q_{T}\right)}^{2} \leq C\left\|\exp \left(\beta \int_{x_{n}}^{1} \frac{d \tau}{\varphi(\tau)}\right) \varphi^{\gamma}\left(x_{n}\right) f\right\|_{L_{2}\left(Q_{T}\right)}^{2} .
$$

Moreover, if $f_{t^{k}} \in H_{\beta, \gamma}^{h}\left(Q_{T}\right), k \leq 2+h$, and $f_{t^{k}}(x, 0)=0$ for $k=0,1, \ldots, 1+h$, then $u \in H_{\beta, \gamma}^{2+h}\left(Q_{T}\right)$ and satisfies

$$
\|u\|_{H_{\beta, \gamma}^{2+h}\left(Q_{T}\right)}^{2} \leq C \sum_{k=0}^{2}\left\|f_{t^{k}}\right\|_{H_{\beta, \gamma}^{h}\left(Q_{T}\right)}^{2}
$$

For the two-dimensional case $(n=2)$, and letting $\varphi(\tau)=\tau^{2}$, we consider problem (5.2) in the cylinder $Q_{T}=\Omega \times(0, T)$, where $\Omega$ is a bounded domain in $\mathbb{R}^{2}, \partial \Omega \backslash\{O\}$ is smooth, and

$$
\{(x, y) \in \Omega: 0<x<1\} \equiv\left\{(x, y) \in \mathbb{R}^{2}: 0<x<1,|y|<x^{2}\right\} .
$$

Thus, the change of variables

$$
\xi=\int_{x}^{1} \frac{d \tau}{\tau^{2}}=x^{-1}-1, \quad \eta=y x^{-2}
$$

transforms

$$
\{(x, y) \in \Omega: 0<x<1\} \text { on to } \mathcal{C}_{+}:=\{(\xi, \eta): \xi>0, \eta \in(-1,1)\} \text {. }
$$


With notations $v(\xi, \eta, t)=u(x, y, t)$, we have

$$
\begin{gathered}
u(x, y)=v\left(x^{-1}-1, y x^{-2}\right), \\
\partial_{y} u=x^{-2} \partial_{\eta} v, \quad \partial_{y y}^{2} u=x^{-4} \partial_{\eta \eta}^{2} v, \quad \partial_{x} u=-x^{-2} \partial_{\xi} v-2 y x^{-3} \partial_{\eta} v \\
\partial_{x x}^{2} u=x^{-3} \partial_{\xi} v+6 y x^{-4} \partial_{\eta} v+x^{-4} \partial_{\xi \xi}^{2} v+4 y x^{-5} \partial_{\xi \eta}^{2} v+4 y^{2} x^{-6} \partial_{\eta \eta}^{2} v \\
=x^{-4}\left[x \partial_{\xi} v+6 y \partial_{\eta} v+\partial_{\xi \xi}^{2} v+4 y x^{-1} \partial_{\xi \eta}^{2} v+4 y^{2} x^{-3} \partial_{\eta \eta}^{2} v\right] \\
=x^{-4}\left[\partial_{\xi \xi}^{2} v+4 \eta(\xi+1)^{-1} \partial_{\xi \eta}^{2} v+4 \eta^{2}(\xi+1)^{-2} \partial_{\eta \eta}^{2} v\right. \\
\left.+(\xi+1)^{-1} \partial_{\xi} v+6 \eta(\xi+1)^{-2} \partial_{\eta} v\right] .
\end{gathered}
$$

Hence, the differential operator $\widehat{\Delta}$, which arises from the differential operator $x^{4} \Delta u$ via the coordinate change $(x, y) \rightarrow(\xi, \eta)$, turns out to be

$$
\begin{aligned}
\widehat{\Delta} v= & \partial_{\xi \xi}^{2} v+\partial_{\eta \eta}^{2} v+4 \eta(\xi+1)^{-1} \partial_{\xi \eta}^{2} v+4 \eta^{2}(\xi+1)^{-2} \partial_{\eta \eta}^{2} v \\
& +(\xi+1)^{-1} \partial_{\xi} v+6 \eta(\xi+1)^{-2} \partial_{\eta} v .
\end{aligned}
$$

Clearly, coefficients of differential operator $\widehat{\Delta}$ stabilize for $\xi \rightarrow+\infty$, and the limit differential operator of $\widehat{\Delta}$ (denoted by $\widehat{\Delta}$ for convenience) is

$$
\widehat{\Delta} v=\partial_{\xi \xi}^{2} v+\partial_{\eta \eta}^{2} v
$$

We denote also by $\mathfrak{U}(\lambda)(\lambda \in \mathbb{C})$, the operator corresponding to the parameter-depending boundary value problem

$$
\widehat{v}_{\eta \eta}+\lambda^{2} \widehat{v}=0, \quad \widehat{v}(-1)=\widehat{v}(1)=0 .
$$

Eigenvalues of $\mathcal{U}(\lambda)$ are roots of the Bessel function

$$
J_{-1 / 2}(z)=\left(\frac{2}{\pi z}\right)^{1 / 2} \cos z
$$

$J_{v}, v>-1$ has only real roots (see [10, Theorem 1, page 94]). Therefore, they are

$$
\lambda_{k}=\frac{\pi}{2}+k \pi, \quad k \in \mathbb{Z}
$$

It is easy to see that $\lambda_{+}=\pi / 2$ is the least positive root of the Bessel function $J_{-1 / 2}(z)$. From arguments above in combination with Theorems 4.6 and 4.7, we obtain the following results: 
Theorem 5.1. Suppose that $f_{t^{k}} \in H_{\beta, \gamma}^{0}\left(Q_{T}\right), k \leq 2,|\beta|<\pi / 2, \gamma$ is a real number and $f_{t^{k}}(x, 0)=0$, for $k=0,1$. Then problem (5.2) has a unique solution $u$ in $H_{\beta, \gamma}^{2}\left(Q_{T}\right)$, and we have the estimate

$$
\|u\|_{H_{\beta, \gamma}^{2}\left(Q_{T}\right)}^{2} \leq C \sum_{k=0}^{2}\left\|e^{\beta(1 / x-1)} x^{2 \gamma} f_{t^{k}}\right\|_{L_{2}\left(Q_{T}\right)}^{2}
$$

Moreover, if $f_{t^{k}} \in H_{\beta, \gamma}^{h}\left(Q_{T}\right), k \leq 2+h$, and $f_{t^{k}}(x, 0)=0$ for $k=0,1, \ldots, 1+h$, then $u \in H_{\beta, \gamma}^{2+h}\left(Q_{T}\right)$ and satisfies

$$
\|u\|_{H_{\beta, r}^{2+h}\left(Q_{T}\right)}^{2} \leq C \sum_{k=0}^{2}\left\|f_{t^{k}}\right\|_{H_{\beta, \gamma}^{h}\left(Q_{T}\right)}^{2}
$$

In case that boundary of $\Omega$ has some cuspidal points, then by arguments analogous to Section 4 , we consequently obtain the similar results.

\section{Acknowledgment}

This work was supported by National Foundation for Science and Technology Development (NAFOSTED), Vietnam.

\section{References}

[1] N. M. Hung, "The first initial boundary value problem for Schrödinger systems in non-smooth domains," Differentsial'nye Uravneniya, vol. 34, pp. 1546-1556, 1998 (Russian).

[2] N. M. Hung, "Asymptotic behavior of solutions of the first boundary value problem for strongly hyperbolic systems near a conical point of the domain boundary," Matematicheskiu Sbornik, vol. 190, no. 7, pp. 103-126, 1999.

[3] N. M. Hung and N. T. Anh, "Regularity of solutions of initial-boundary value problems for parabolic equations in domains with conical points," Journal of Differential Equations, vol. 245, no. 7, pp. 18011818, 2008.

[4] N. M. Hung and J.-C. Yao, "On the asymptotics of solutions of the first initial boundary value problem to hyperbolic systems in infinite cylinders with base containing conical points," Nonlinear Analysis: Theory, Methods \& Applications, vol. 71, no. 5-6, pp. 1620-1635, 2009.

[5] N. M. Hung and V. T. Luong, "Unique solvability of initial boundary-value problems for hyperbolic systems in cylinders whose base is a cusp domain," Electronic Journal of Differential Equations, vol. 2008, no. 138, pp. 1-10, 2008.

[6] V. T. Luong, "On the first initial boundary value problem for strongly hyperbolic systems in nonsmooth cylinders," Journal of S. HNUE, vol. 1, no. 1, 2006.

[7] V. A. Kozlov, V. G. Maz'ya, and J. Rossmann, Elliptic Boundary Value Problems in Domains with Point Singularities, vol. 52 of Mathematical Surveys and Monographs, American Mathematical Society, Providence, RI, USA, 1997.

[8] V. G. Maz'ya and B. A. Plamenevskiř, "Estimates in $L_{p}$ and in Hölder classes, and the Miranda-Agmon maximum principle for the solutions of elliptic boundary value problems in domains with singular points on the boundary," Mathematische Nachrichten, vol. 81, pp. 25-82, 1978, English translation in: American Mathematical Society Translations, vol. 123, pp. 1-56, 1984.

[9] L. C. Evans, Partial Differential Equations, vol. 19 of Graduate Studies in Mathematics, American Mathematical Society, Providence, RI, USA, 1998.

[10] B. G. Korennev, Bessel Functions and Their Applications, Chapman \& Hall/CRC Press, Boca Raton, Fla, USA. 